\title{
ReVieW of Real Time EFficient Web Page SEARChes BaSed on RANKING
}

\author{
S. HASEENA ${ }^{1 *}$, R. LATHA ${ }^{2}$, A.M. BARANI ${ }^{3}$ \\ ${ }^{1 *}$ Department of Computer Science \& Application, St.Peter's Institute of Higher Education and Research, Chennai, India \\ ${ }^{2}$ Department of Computer Science \& Application, St.Peter's Institute of Higher Education and Research, Chennai, India \\ ${ }^{3}$ Department of Computer Science \& Application, St.Peter's Institute of Higher Education and Research, Chennai, India
}

Available online at: www.isroset.org

Received: 18/Mar/2018, Revised: 26/Mar/2018, Accepted: 02/Apr/2018, Online: 30/Apr/ 2018

\begin{abstract}
Web surfer are explicitly intended for looking website pages, archives and pictures were created to encourage searching through a vast, amorphous blob of unstructured assets. They are built to take after a multi-organize process: creeping the vast reserve of pages and archives to glance through at once in the allegorical froth from their substance, ordering the froth/popular expressions in a kind of semi-organized frame (database or something), and finally, settling client sections/questions to return most precise or logical relevance to the outcomes and connections to those filtered records or pages from the stock.
\end{abstract}

Keywords- look interface, crawler,indexer, and database

\section{INTRODUCTION}

An internet searcher is a data recovery programmer that finds, creeps, changes and stores data for recovery.[ Christian Quast et al]A web search tool ordinarily comprises of four parts e.g. look interface, crawler (otherwise called a bug or bot), indexer, and database[9]. The crawler crosses a record accumulation, deconstructs archive message, and allocates surrogates for capacity in the internet searcher list. Online web crawlers store pictures interface information and metadata for the record. This process is called Web crawling or spidering. Many sites, in particular search engines, use spidering as a means of providing up-to-date data. Web crawlers are mainly used to create a copy of all the visited pages for later processing by a search engine that will index the downloaded pages to provide fast searches. Crawlers can also be used for automating maintenance tasks on a Web site, such as checking links or validating HTML code. Also, crawlers can be used to gather specific types of information from Web pages, such as e-mail addresses. A Web crawler is one type of software agent. In general, it starts with a list of URLs to visit, called the seeds. As the crawler visits these URLs, it identifies all the hyperlinks in the page and adds them to the list of URLs to visit, called the crawl frontier. URLs from the frontier are recursively visited according to a set of policies.

\section{Process of Web Page Searches}

The idea of hypertext and a memory expansion begins from an article that was distributed in The Atlantic Monthly in July 1945 composed by Vannevar Bush, titled As We May Think. Inside this article, Vannevar encouraged researchers to cooperate to help construct an assortment of information for all humankind. He at that point proposed the possibility of a for all intents and purposes boundless, quick, solid, extensible, cooperative memory stockpiling and recovery framework. He named this gadget a memex. [8]

Shrubbery respected the thought of acquainted ordering as his key theoretical commitment. As he clarified, this was an arrangement whereby any thing might be caused voluntarily to choose instantly and naturally another. This is the basic component of the memex. The procedure of entwining two things is the imperative thing. This connecting (as we now say) constituted a trail of reports that could be named, coded, and discovered once more. In addition, after the first two things were coupled, various things could be combined to shape a trail, they could be investigated thus, quickly or gradually, by redirecting a lever like that utilized for turning the pages of a book. It is precisely as if the physical things had been assembled from broadly isolated sources and bound together to frame another book [3]. 


\section{Existing Method of Web Page Searches}

\section{A. Conventional search}

The idea of archive has been characterized as any solid or emblematic sign, safeguarded or recorded, for reproducing or for demonstrating a wonder, regardless of whether physical or mental. [4]

The developing thought of the archive among Jonathan Priest, Otlet, Briet, Schürmeyer, and alternate documentalists progressively stressed whatever worked as a report instead of conventional physical types of records. The move to computerized innovation would appear to make this refinement considerably more critical. Collect's insightful investigations have demonstrated that an accentuation on the innovation of advanced archives has obstructed our comprehension of computerized records as reports [8]. A customary record, like a mail message or a specialized report, exists physically in advanced innovation as a series of bits, as does everything else in a computerized situation. As a question of study, it has been made into an archive. It has turned out to be physical proof by the individuals before making a decision or reaching a conclusion.

\section{B. Text Based Search}

In content recovery, full-content scan alludes to strategies for looking through a solitary PC put away archive or a gathering in a full-content database. The full-content inquiry is recognized from looks in light of metadata or on parts of the first messages stored in databases, (for example, titles, abstracts, chose segments, or bibliographical references).

In a full-content pursuit, a web search tool analyzes the majority of the words in each put-away archive as it tries to coordinate hunt criteria (for instance, content indicated by a client). Full-content seeking systems wound up plainly normal in online bibliographic databases in the 1990s. Numerous sites and application programs, (for example, word preparing to the programme) give full-content hunt capacities. Some web crawlers, for example, AltaVista, utilize fullcontent pursuit methods, while others record just a segment of the pages analyzed by their ordering systems. [4]

\section{Multimedia Search}

Mixed media look empowers data to seek to utilize inquiries in numerous information sorts including content and other interactive media designs. Sight and sound hunt can be actualized through multimodal look interfaces, i.e., interfaces that permit submitting seek questions as printed asks for as well as through other media [5].

Pursuit is made utilizing the layers in metadata which contain data of the substance of a sight and sound record. Metadata look is less demanding, quicker and successful in light of the fact that as opposed to working with the unpredictable material, for example, a sound, a video or a picture, it seeks utilizing content.

\section{Conceptual Search}

An idea seek (or calculated pursuit) is a computerized data recovery strategy that is utilized to look electronically put away unstructured content (for instance, advanced documents, email, logical writing, and so on.) for data that is adroitly like the data gave in a hunt question[2]. As it were, the thoughts communicated in the data recovered in light of an idea seek inquiry are significant to the thoughts contained in the content of the question.

Idea seek procedures were produced in view of impediments forced by established Boolean watchword look innovations when managing extensive, unstructured advanced accumulations of content. Catchphrase seeks regularly return comes about that incorporate numerous non-important things (false positives) or that reject an excessive number of pertinent things (false negatives) on account of the impacts of synonymy and polysemy[4]. Synonymy implies that one of at least two words in a similar dialect have a similar significance, and polysemy implies that numerous individual words have more than one importance.

\section{E. Information System Search}

Data Systems Research is an associate inspected scholarly diary that spreads inquire about in the territories of data frameworks and data innovation, including subjective brain research, financial aspects, software engineering, operations look into, plan science, association hypothesis and conduct, human science, and key administration. It is distributed by the Institute for Operations Research and the Management Science and was as of late chosen as one of the main 20 proficient/scholastic diaries by BusinessWeek.[4] Along with Management Information Systems Quarterly, Information Systems Research is viewed as one of the two most renowned diaries in the data frameworks discipline.[6][7]

\section{F. Personalised Search}

Customized seek suggests web look encounters that are custom-made particularly to a person's advantages by joining data about the person past particular question gave. There are two general ways to deal with customizing list items, one including adjusting the client's question and the other repositioning hunt results. [10]

\section{G. Page Rank}

PageRank is a connection investigation calculation and it allows a numerical weighting to every component of a hyperlinked set of reports, for example, the World Wide Web, with the motivation behind measuring its relative significance 
inside the set. The calculation might be connected to any gathering of substances with complementary citations and references. The numerical weight that it allows to any given component $\mathrm{E}$ is alluded to as the PageRank of $\mathrm{E}$ and meant by PR (E). Different elements like Author Rank can add to the significance of an element.

A PageRank comes about because of a scientific calculation in view of the web diagram, made by all World Wide Web pages as hubs and hyperlinks as edges, thinking about expert centers, for example, cnn.com or usa.gov. The rank esteem demonstrates a significance of a specific page. A hyperlink to a page considers a vote of help. The PageRank of a page is characterized recursively and relies upon the number and PageRank metric of all pages that connect to it (approaching connections). A page that is connected to by many pages with high PageRank gets a high rank itself.

\section{H. Ranking (Information Retrieval)}

The positioning of inquiry comes about is one of the basic issues in data recovery (IR), the logical/building discipline behind web indexes. Given a question and answer gathering $\mathrm{D}$ of records that match the inquiry, the issue is to rank [10], that is, sort, the reports in D as indicated by some model so that the best results seem right on time in the outcome list showed to the client. Traditionally, positioning criteria is relating directly and significantly of records to be communicated in the inquiry.

Positioning capacities are assessed by different ways. one of the least difficult is deciding the exactness of the main $\mathrm{k}$ toppositioned[1] comes about for some settled k.For instance, the extent of the best 10 comes about that are important, by and large, finished many inquiries.

Figuring out how to rank[4] or machine-picked up positioning (MLR) is the use of machine adapting, ordinarily directed, semi-administered or support learning, in the development of positioning models for data recovery systems.[8] Training information comprises of arrangements of things with some fractional request determined between things in each rundown. This request is commonly actuated by giving a numerical or ordinal score or a double judgment (e.g. pertinent or not important) for everything. The positioning model's motivation is to rank, i.e. deliver a stage of things in new, inconspicuous records in a way which is comparative to rankings in the preparation information in some sense.

\section{Computing Search.}

A web search tool is a data recovery framework intended to help discover data put away on a PC framework. The list items are typically exhibited in a rundown and are usually called hits. Web search tools help to limit the time required to discover data and the measure of data which must be counseled, similar to different strategies for overseeing data over-burden.

The most open, noticeable type of a web search tool is a Web internet searcher which looks for data on the World Wide Web.

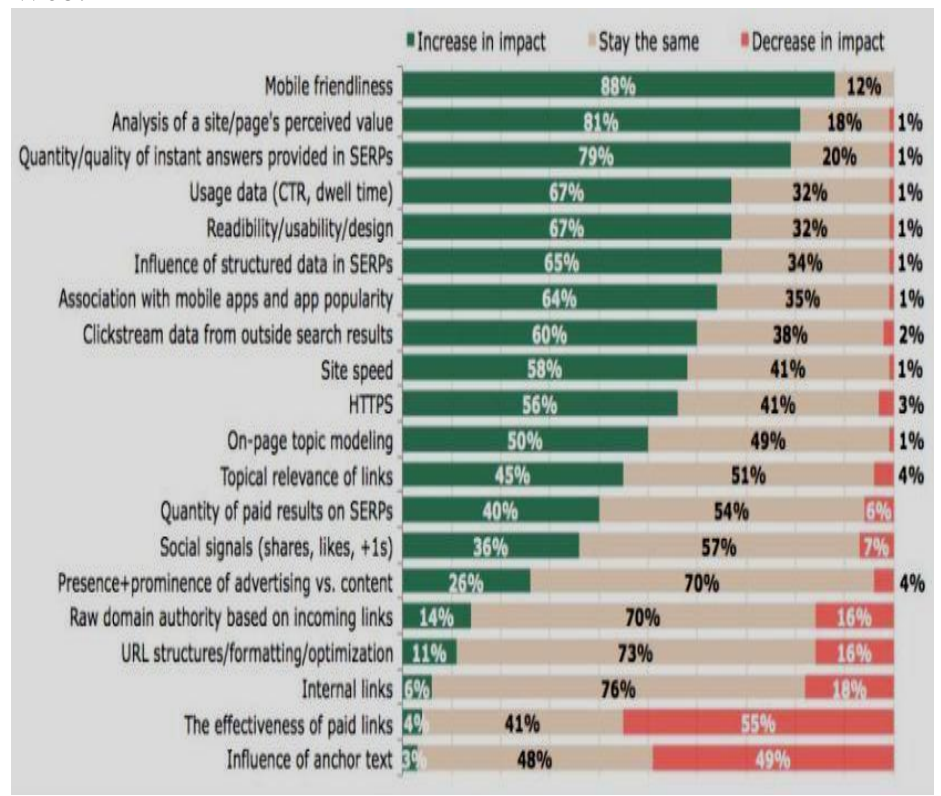

Figure.1 Impact changes of Search and ranking

\section{Conclusion and Future Scope}

On account of an entire literary hunt, the initial phase in grouping of website pages is to discover a list of thing that may relate explicitly to the pursuit term. Earlier, web indexes started with a little rundown of URLs as a purported seed list, brought the substance, and parsed the connections on those pages for pertinent to data, which gave new connections. The procedure was very patterned and preceded until the point when enough pages were found for the searcher's utilization. Nowadays, a constant creep strategy is utilized rather than an accidental revelation in light of a seed list. The creep technique is an augmentation of previously mentioned revelation strategy. But there is no seed list on the grounds that the framework works constantly.

Most web crawlers utilize modern planning calculations to choose when to return to a specific page, to engage its pertinence. WebCrawler evolved to accommodate the extraordinary growth of the Web. This growth affected WebCrawler not only by increasing the size and scope of its components had to change to accommodate this growth: the crawler had to download more documents, the full-text index had to become more efficient at storing and finding those documents, and the Service had to accommodate heavier demand. Such changes were not only related to scale, however: the evolving nature of the Web meant that functional changes were necessary, too, such as the ability to 
handle raw queries from searchers.

Index, but also by increasing the demand for its service. Each of WebCrawler's These calculations run from steady visitinterim with higher need for all possible changing pages to versatile visit-interim in light of a few criteria. For example, recurrence of progress, fame, and general nature of the site. The speed of the web server running the page and also set limitations like the measure of equipment or data transfer capacity.

\section{REFERENCES}

[1] Adriaanse L and Rensleigh C (2013). Web of Science, Scopus and Google Scholar. The Electronic Library, 31(6), 727-744.

[2] Aniko Hannak, Piotr Sapiezynski, Arash Molavi, Kakhki, Balachander Krishnamurthy, David Lazer, Alan Mislove and Christo Wilson (2013)"Measuring Personalization of Web Search (PDF)" Archived from the original (PDF) on April 25, 2013.

[3] Bornmann, L, Leydesdorff L and Mutz R (2013). The use of percentiles and percentile rank classes in the analysis of bibliometric data: Opportunities and limits. Journal of Informetrics, 7(1), 158-165.

[4] D. Coltuc and J.-M. Chassery, "Very fast watermarking by reversible contrast mapping," IEEE Signal Process. Lett., vol. 14, no. 4, pp. 255 258, Apr. 2007. Christian Quast, Elmar, Pruesse Pelin, Yilmaz Jan, Gerken Timmy, Schweer Pablo, Yarza Jörg, Peplies Frank and Oliver Glöckner (2013) " The SILVA ribosomal RNA gene database project: improved data processing and web-based tools" at Nucleic Acids Research, Volume 41, Issue D1, 1 January 2013, Pages D590-D596.

[5] Jayanthi.J and Dr.K.S.Jayakumar (2011) "An integrated Page Ranking Algorithm for Personalized Web Search". In International Journal of Computer Applications (0975- 8887), Volume 12-No.11, January 2011.

[6] Manikandan, R and Dr. R.Latha (2017).“A literature survey of existing map matching algorithm for navigation technology," International journal of Engineering Sciences \& Research Technology”, 6(9), 326331.Retrieved September 15, 2017.

[7] Mercy paul Selvan, A . Chandra Sekar and A. Priya Dharshini (2012) "Survey on Web page Ranking Algorithms"at International Journal of Computer Applications, Vol.41, No-19, ISSN 0975-8887.

[8] Northcott, D. and Linacre S (2010). Producing spaces for academic discourse:The impact of research assessment exercises and journal quality rankings. Australian Accounting Review, 20(1), 38- 54.

[9] Sergey Brin and Lawrence Page (2012) "The anatomy of large scale hypertexual search engine" at Computer Networks Elsevier, vol.56, issue-18, pp3825-3833.

[10] Mitsuo Yokokawa, Fumiyoshi Shoji, Atsuya Uno, Motoyoshi Kurokawa and Tadashi Watanabe (2011). The K computer: Japanese next-generation supercomputer development project. International Symposium on Low Power Electronics and Design (ISLPED). pp. 371-372. doi:10.1109/ISLPED.2011.5993668 (1-3 August 2011).
Authors Profile

Mrs.S.Haseena pursed Bachelor of Computer Application from Thiruvalluvar University in 2009 and Master of Information Technology from Madras University in 2011 and M.Phil. From Sankara University in 2014. She is currently pursuing Ph.D. and currently working in Guest Lecturer in Department of Computer Science. She has published more than 6 research papers in reputed international journals including and conferences including IEEE and it's also available online. Her main research work focus on Data Mining and Big Data analysis. She has 6 years of teaching experience and 3 years of Research Experience.

Dr.R.Latha, pursed Bachelor of Science (Maths) from Bharathidasan University in year 1986 and Master of Applicable Maths \& Computer Science from Bharathidasan University in year 1988 and M.Phil. from Bharathidasan University in Year 2004, Master of Computer Application from Periyar

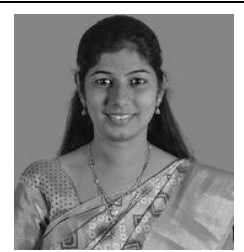
University in Year 2010and M.E from St. Peter's University in year 2014and Ph.D. from Dr.MGR University in 2011. She is currently working Head of the Department of Computer Science, St. Peter's University, Avadi. Her main research work focus on Data Mining and Data Ware Housing. She has published more than 29 research papers in reputed international journals including and 13 International conference and 102 National Conference including IEEE and it's also available online. She has more than 20 years of teaching experience and more than 10 years of Research Experience.

Mrs.A.M.Barani pursed Bachelor of Computer Science from Bharadiar University in year 1995 and Master of Computer Application from Madras University, Chennai in year 2000 and M.Phil. From St. Peter's University in year 2014. She is currently pursuing $\mathrm{Ph} . \mathrm{D}$. and currently working as Guest Lecturer in Department of Computer Science,

Thiruvalluvar University of Arts and Science College, Attupakkam. Her main research work focus on Data Mining. She has published more than 2 research papers in reputed international journals including and conferences including IEEE and it's also available online. Her main research work focus on Data Mining. She has 4 years of teaching experience and 3 years of Research Experience. 